\begin{tabular}{|c|c|}
\hline & Number (\%) \\
\hline Total cases & 36 \\
\hline Total patients & 32 \\
\hline Age (years) & $57.9 \pm 13.7$ \\
\hline Male & $5(13.9)$ \\
\hline Total aneurysms treated & 40 \\
\hline Aneurysm size, average & $6.9 \mathrm{~mm}$ \\
\hline Anterior circulation & $38(95)$ \\
\hline Cavernous & $5(12.5)$ \\
\hline Clinoidal & $4(10.0)$ \\
\hline Ophthalmic & $13(32.5)$ \\
\hline Communicating & $8(20.0)$ \\
\hline Anterior communicating & $6(15.5)$ \\
\hline Distal ACA & $2(5)$ \\
\hline Posterior circulation & $2(10)$ \\
\hline PICA & $1(2.5)$ \\
\hline Vertebral & $1(2.5)$ \\
\hline Triaxial system & 36 \\
\hline \multicolumn{2}{|l|}{ Guide sheath } \\
\hline NeuronMax & $30(83.3)$ \\
\hline \multicolumn{2}{|l|}{ Select catheter } \\
\hline JB-1 & $31(86.1)$ \\
\hline \multicolumn{2}{|l|}{ Guide catheter } \\
\hline Catalyst 058 & $36(100)$ \\
\hline \multicolumn{2}{|l|}{ Microcatheter } \\
\hline Marksman 0.027 inch & $3(8.3)$ \\
\hline Via 0.027 inch & $34(94.4)$ \\
\hline Other catheters & $11(30.6)$ \\
\hline Pipeline embolization devices (33 cases, 32 patients) & 40 \\
\hline WEB devices (2 cases) & 2 \\
\hline Coils (3 cases) & 32 \\
\hline Cervical tortuosity & $15(41.2 \%)$ \\
\hline \multicolumn{2}{|l|}{ Guide catheter position } \\
\hline Cervical ICA & $0(0)$ \\
\hline Petrous ICA & $1(2.7)$ \\
\hline Proximal cavernous ICA & $6(16.7)$ \\
\hline Distal cavernous/clinoidal ICA & $24(66.7)$ \\
\hline Ophthalmic & $1(2.7)$ \\
\hline Communicating & $1(2.7)$ \\
\hline ACA & $1(2.7)$ \\
\hline Distal vertebral & $2(5.4)$ \\
\hline \multicolumn{2}{|l|}{ Clinical success } \\
\hline Catalyst tracked to target & $36(100)$ \\
\hline Successful treatment & $36(100)$ \\
\hline
\end{tabular}

patients received a total of 40 pipeline embolization devices (Table 2), 2 received the WEB embolization device, and 3 patients received coils. The Catalyst was used with the Marksman microcatheter in $3(8.3 \%)$ cases, and the VIA microcatheter in $34(94.4 \%)$ cases. The Catalyst was successfully advanced in all cases (Figure 2), with the distal tip located in the petrous ICA 1 (2.7\%), proximal cavernous 6 (16.7), distal cavernous/clinoidal 24 (66.7\%), ophthalmic 1 (2.7\%), communicating 1 (2.7), distal ACA 1 (2.7\%), and distal vertebral artery $2(5.4 \%)$. No patients experienced iatrogenic vessel dissection or other complications related to the catheter.

Conclusions The Catalyst 5 is an adept distal access catheter for cerebral aneurysm embolization with facile utility in the deployment of PEDs, WEB devices, as well as traditional

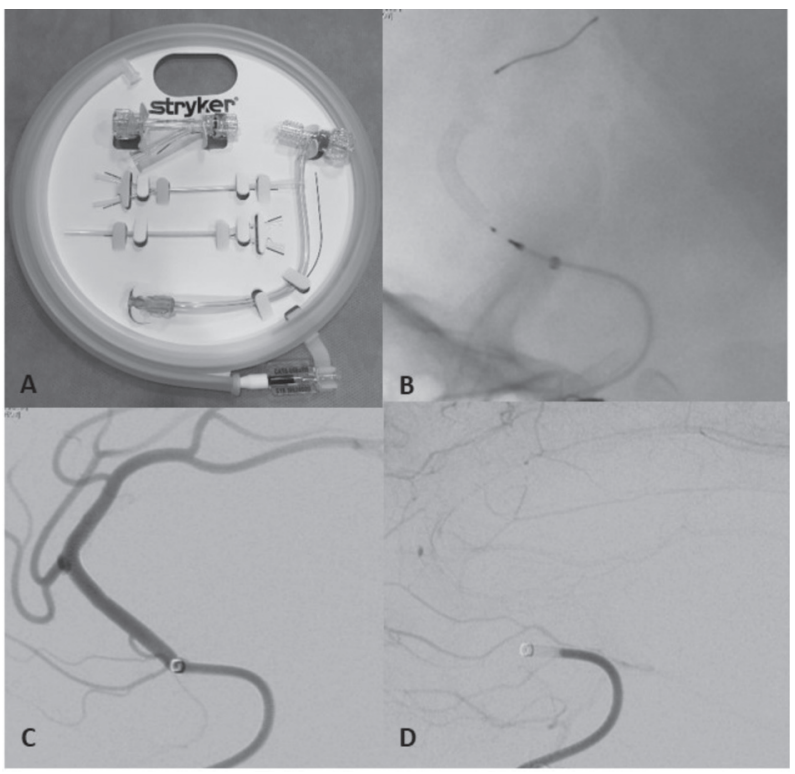

Abstract E-097 Figure 1 (A) The Catalyst5 distal access catheter. (B) Pipeline embolization of an $2.7 \mathrm{~mm} \mathrm{~L} \mathrm{A2-A3} \mathrm{aneurysm.} \mathrm{The} \mathrm{FlexPED} \mathrm{is}$ in the A2/A3 junction with the VIA and Catalyst catheters. (C) Good distal ACA filling without vasospasm after PED deployment. (D) Contrast stasis after deployment.

aneurysm coiling. This catheter has outstanding trackability and stability during device deployment, and is a useful option to have when achieving distal access and support in an atraumatic fashion in the treatment of intracranial aneurysms.

Disclosures G. Colby: None. L. Lin: None. R. Xu: None. M. Bender: None. B. Jiang: None. D. Lubelski: None. A. Coon: None.

\section{E-098 COMPARE THE PROGNOSTIC VALUE BETWEEN DIFFUSION VOLUME AND PERFUSION/DIFFUSION MISMATCHING}

${ }^{1}$ Y. Won, ${ }^{2} \mathrm{D}$ Yoo, ${ }^{2} \mathrm{~T}$ Won, ${ }^{3} \mathrm{~S}$ Lee. ${ }^{1}$ Radiology, Uijeongbu St. Mary's Hospital, Uijeongbu-si, Korea, Republic of; ${ }^{2}$ Neurosurgery, Uijeongbu St. Mary's Hospital, Uijeongbu-si, Republic of Korea; ${ }^{3}$ Neurology, Uijeongbu St. Mary's Hospital, Uijeongbu-si, Republic of Korea

\subsection{6/neurintsurg-2016-012589.170}

Background and purpose Diffusion (DWI) change area regard as cytotoxic edema and ischemic core. And perfusion/diffusion mismatching ( $\mathrm{P} / \mathrm{D}$-mismatch) is regard as ischemic penumbra and targeted tissue to ischemic stroke treatment. Exact diffusion volume may be important to speculate the patient prognosis. But there were no studies which compare the clinical significance of the diffusion volume and P/D-mismatch.

Materials and methods 57 patients whom treated additional IA-Tx, non-recanalised after IV-tPA with anterior circulation and major vessel occlusion, were analyzed retrospectively. Diffusion volume was calculated from MR graphic program and $\mathrm{P} / \mathrm{D}$-mismatch was evaluated by radiologist who was not involved in patient treatment. Statistical analysis were done according to the DWI volume and $\mathrm{P} / \mathrm{D}$-mismatch, in recanalization, favorable outcome, and significant hemorrhage.

Results $\mathrm{P} / \mathrm{D}$-mismatch was statistical significant prospect on favorable outcome $\left(\chi^{2}, \mathrm{p}=0.000\right)$, neuroloic improvement $\left(\chi^{2}, \mathrm{p}=0.000\right)$, significant hemorrhage $\left(\chi^{2}, \mathrm{p}=0.043\right)$, 
extravascation $\left(\chi^{2}, \mathrm{p}=0.000\right)$ and decompressive surgical incidence $\left(\chi^{2}, \mathrm{p}=0.007\right)$. But diffusion volume, evaluated cording to $30 \mathrm{cc}, 60 \mathrm{cc}, 100 \mathrm{cc}$ grading analysis, not predict neurologic outcomes, hemorrhagic complications.

Conclusion In this study, diffusion volume calculation is impossible to calculate without computerized program and clinical significance of diffusion volume was questionable. P/Dmismatch was more significant prognostic indicator than diffusion volume in acute stroke patients management.

Disclosures Y. Won: None. D. Yoo: None. T. Won: None. S. Lee: None.

\section{\begin{tabular}{|l|l}
\hline E-099 DOES ANTERIOR CEREBRAL ARTERY VASOSPASM \\
\hline
\end{tabular} AFTER SPONTANEOUS SUBARACHNOID HEMORRHAGE PREDICT SHORT TERM COGNITIVE OUTCOME}

${ }^{1}$ V Pandav, ${ }^{2} \mathrm{~B}$ Bohnstedt, ${ }^{3} \mathrm{~L}$ Yearout, ${ }^{4} \mathrm{D}$ Thompson, ${ }^{1} \mathrm{~B}$ Ray. ${ }^{1}$ Neurology, The University of Oklahoma Health Sciences, Oklahoma City, OK; ${ }^{2}$ Neurosurgery, The University of Oklahoma Health Sciences, OKlahoma City, OK; ${ }^{3}$ Biostatics and Epidemiology, The University of Oklahoma Health Sciences, Oklahoma City, OK; ${ }^{4}$ Biostatistics and Epidemiology, The University of Oklahoma Health Sciences, Oklahoma City, OK

\subsection{6/neurintsurg-2016-012589.171}

Background Spontaneous subarachnoid hemorrhage (SAH) is a disabling form of hemorrhagic stroke that affects young individuals and is responsible for short-term and long-term cognitive deficits. Delayed cerebral ischemia (DCI) is postulated to be the major determinant for this morbidity. Cerebral vasospasm (CVS) is a major contributor of DCI. Treatment of symptomatic CVS can consist of intra-arterial vasodilators or angioplasty. This study reports preliminary data on cognitive outcomes in a prospective cohort of patients with spontaneous $\mathrm{SAH}$ who received vasodilator therapy for anterior cerebral artery (ACA) vasospasm.

Aim

1. Determine role of delayed cerebral ischemia (DCI) and CVS after spontaneous SAH on short-term cognitive outcome.

2. Determine if ACA vasospasm predicts short-term cognitive outcome.

3. Determine if treatment of ACA vasospasm results in improvement in short-term cognitive outcome.

Methods Thirty-five patients with clinical follow-up at 3 months after $\mathrm{SAH}$ were selected from a prospective cohort. DCI was defined as a new hypodensity on CT scans located in a vascular territory, with or without symptoms (decrease of consciousness or focal deficits), due to CVS and not explained by other causes (e.g. rebleeding, hydrocephalus, cardioembolic sources, hypoxia, electrolyte disturbances, or seizures). CVS was defined as $\geq 25 \%$ narrowing on digital subtraction angiogram (aCVS) or Transcranial Doppler mean flow velocity $\geq 120 \mathrm{~cm} / \mathrm{sec}$ (sCVS). Cognitive outcomes were assessed using Montreal Cognitive Outcome Assessment (MOCA) and poor cognitive outcome was defined as MOCA score <26. Fisher's exact tests and logistic regression were performed to analyze the study questions.

Results Average age of the study cohort was $53.1 \pm 11.5$ years with $71 \%$ of the patients being women. DCI occurred in $16 / 35(45.7 \%)$ patients. In the absence of sCVS, DCI predicted poor cognitive outcomes (3/5 with DCI, $60 \%$ vs $1 / 10$ without DCI, $10 \% ; \mathrm{p}=0.04)$. Patients with anterior cerebral artery or anterior communicating complex (ACA/ACom) related aneurysmal SAH were less likely to have MOCA $<26$
(ACA/ACom vs others $\quad(3 / 12, \quad 25 \%$ vs $11 / 23, \quad 47.8 \%$; $\mathrm{p}=0.28)$. Of the 22 patients who underwent digital subtraction angiogram for clinical indication, 10 were found to have ACA CVS and were treated. Patients with SAH due to ACA/ ACom location aneurysms displayed low MOCA scores less often than those with SAH in other locations $(2 / 5 ; 40 \%$ vs 4 / $5 ; 80 \% ; \mathrm{p}=0.15)$. A two-factor logistic regression model found that, while holding treatment status constant, the odds of a poor cognitive outcome were 4.6 times higher (90\% CI on odds ratio: 1.0, 21.3; $\mathrm{p}=0.0998$ ) among those with $\mathrm{SAH}$ outside of ACA/ACom aneurysms.

Conclusions Our study reaffirms that occurrence of DCI after $\mathrm{SAH}$ predicts poor cognitive outcome at 3 months. The fact that cognitive outcomes were not superior in patients treated for CVS suggests that a complex pathophysiology determines outcomes after SAH. Relatively poor cognitive outcome among patients with SAH in locations other than ACA/ACom alludes to involvement of functional neural network apart from frontal lobe based networks that needs further investigation.

Disclosures V. Pandav: None. B. Bohnstedt: None. L. Yearout: None. D. Thompson: None. B. Ray: None.

\section{E-100 USE OF THE 0.027 VIA MICROCATHETER FOR PIPELINE EMBOLIZATION OF CEREBRAL ANEURYSMS: A TECHNICAL NOTE}

${ }^{1} \mathrm{~L}$ Lin, ${ }^{2} \mathrm{G}$ Colby, ${ }^{2} \mathrm{R}$ Xu, ${ }^{2} \mathrm{M}$ Bender, ${ }^{2} \mathrm{~B}$ Jiang, ${ }^{2} \mathrm{D}$ Lubelski, ${ }^{2} \mathrm{~A}$ Coon. ${ }^{1}$ Neurosurgery, UC Irvine, Irvine, $C A ;{ }^{2}$ Neurosurgery, Johns Hopkins, Baltimore, MD

\subsection{6/neurintsurg-2016-012589.172}

Introduction Pipeline embolization devices (PEDs) are designed for delivery through a 0.027" microcatheter such as the Marksman (Medtronic). Challenges with second generation FlexPEDs include limited support from the Marksman for consistent resheathing and providing enough push for delivery.

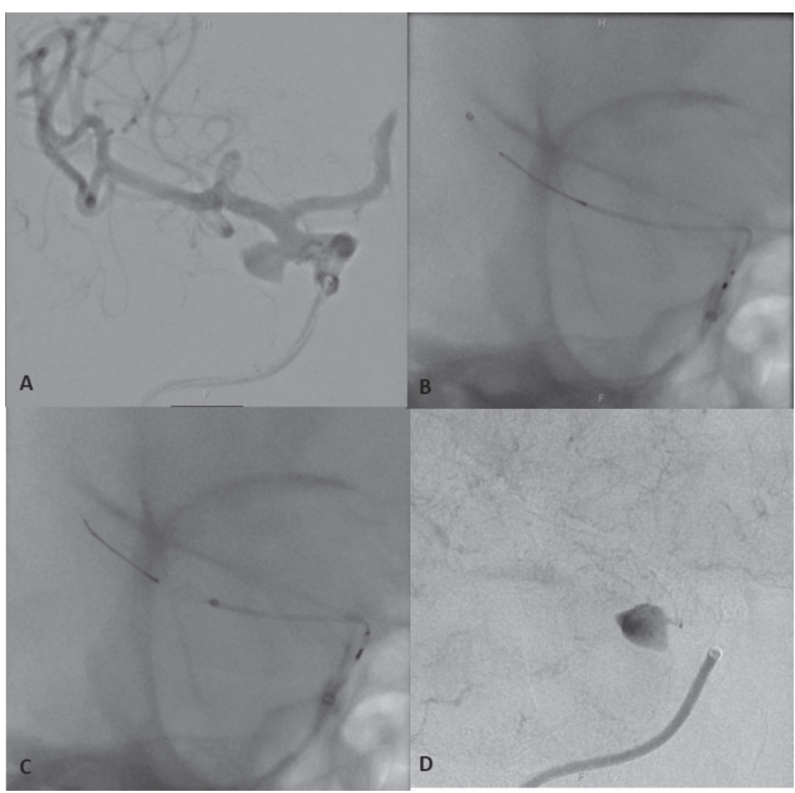

Abstract E-100 Figure 1 (A) Angiogram demonstrates a R Pcomm aneurysm. (B) Introduction of the PED. The distal PED was opened in the MCA and withdrawn back into the supraclinoid ICA. (C)

Deployment of the PED across the aneurysm neck. (D) Post-deployment angiogram shows significant contrast stasis. 